\title{
Branding, un elemento necesario del marketing estratégico en la Cámara de Comercio de Ambato
}

Fecha de recepción: 10/01/2020 • Fecha de aceptación: 15/03/2020 • Fecha de publicación:10/06/2020

Diego Mauricio Bonilla Jurado ${ }^{1}$ Universidad Estatal de Bolívar dbonilla@ueb.edu.ec https://orcid.org/0000-0002-4784-7861

Natalia Del Rocío Delgado Salcedo² Universidad de Especialidades Espíritu Santo natydelgado@hotmail.es https://orcid.org/0000-0002-0297-9035

Graciela Maribel Fajardo Aguilar ${ }^{3}$ Universidad de Machala gfajardo@utmachala.edu.ec https://orcid.org/0000-0003-1152-6414

\section{RESUMEN:}

El objetivo del presente estudio fue analizar la imagen corporativa que percibe la población con respecto a la Cámara de Comercio de Ambato. Para ello se utilizó un enfoque cualitativo, se describió la situación actual de la imagen para conocer su significado, valor, reacción, conocimiento y estética al mostrar el isotipo actual. Se utilizó la fenomenología como técnica para el análisis del discurso en los sujetos de estudio, la muestra comprendió edades entre 20 y 49 años entre hombres y mujeres que trabajan en instituciones del sector público. Los resultados demostraron que la imagen corporativa no es reconocida por la población, luego de indicarles el logotipo completo no la relacionaron con la imagen institucional, tampoco encontraron vinculación de sus servicios, con respecto a la misión, visión y valores corporativos; el logo no lo consideran creativo, con respecto a su slogan se consideró que no es impactante, la mayoría de los encuestados no lo asociaron con el organismo. Se concluyó 
que antes de mejorar la imagen corporativa, la institución debe reconstruir su filosofía empresarial concertando su misión, visión y valores, por la variedad de servicios que oferta la Cámara, por ende, esto producirá un cambio estructural en la dirección estratégica desde su directorio.

PALABRAS CLAVE: branding, dirección imagen corporativa, filosofía empresarial, planeación estratégica.

\begin{abstract}
The objective of this study was to analyze the corporate image that the population perceives with respect to the Ambato Chamber of Commerce. For this, a qualitative approach was used, the current situation of the image was described to know its meaning, value, reaction, knowledge and aesthetics by showing the current isotype. The phenomenology was used as a technique for the analysis of discourse in subjects study, the Ambato population whose ages range from 20 to 49 years, men and women working in public sector institutions. The results showed that the corporate image is not recognized by the population, after indicating the complete logo, they did not relate it to the institutional image, nor did they find any link between their services, with respect to the mission, vision, and corporate values; the logo is not considered creative, with respect to its slogan it was considered not to be shocking, most of the respondents did not associate it with the institution. It was concluded that before improving the corporate image, the institution must rebuild its business philosophy by agreeing on its mission, vision and values, due to the variety of services that the Chamber offers, therefore, this will produce a structural change in the strategic direction from its board of directors.
\end{abstract}

KEYWORDS: branding, corporate image management, business philosophy, strategic planning. 


\section{Introducción}

La imagen corporativa es la referencia que refleja la fotografía de la institución o empresa hacia las personas, esta fotografía que percibe el público indica la naturaleza de los servicios o la razón social y económica que ella desempeña en determinado sector, esto quiere decir que es la idea general que los consumidores tienen sobre las actividades, productos y conducta de determinada institución. Muchos autores describen a la imagen corporativa con otras expresiones como: imagen integral, imagen global, imagen de marca de la empresa, etc., a pesar de esto, el problema no es la cantidad de expresiones, sino la utilización que se hace de ellas, ya que en ciertas ocasiones no se ajustan a la imagen real de una organización, además, se puede definir como una evocación o representación mental que conforma cada individuo, formada por un cúmulo de atributos referentes a la institución; cada uno de estos atributos puede cambiar, y puede coincidir o no con la combinación de atributos ideales de dicho individuo.

La importancia que proyecta la imagen corporativa va mucho más allá de la relevancia en confeccionar un logo o membrete, es la representación que permite de manera directa y visual al proyectar la identidad de una empresa, organismo o institución (Rivadeneira, 2018), por tanto, al ser una representación mental o algo que se manifiesta internamente y que lo forma cada individuo, las empresas deben interesarse en promover en sus planificaciones anuales y destinar un rubro significante a este concepto, sin estar enfocado desde el punto de vista del gasto sino de inversión de sostenibilidad y posicionamiento (Pintado y Sánchez, 2013).

Todo puede afectar a la imagen, de ahí que ésta se forme por un cúmulo de atributos referentes a la compañía. Estos atributos pueden estar relacionados con el precio y la calidad, generalmente los precios altos se relacionan eventualmente con empresas de buena imagen, mientras que los precios bajos no tienen esta asociación, aún cuando el consumidor compre los productos para beneficiarse de esos precios (Pintado y Sánchez, 2013). La importancia que proyecta la imagen corporativa, va mucho más allá de la significación de confeccionar un logo o membrete. Es la representación que permite de manera directa y visual, proyectar la identidad de una empresa, organismo o institución. La imagen corporativa parte de un principio básico en el mundo del marketing que sirve para el establecimiento de una marca para ofrecer productos y servicios (Molina, 2010).

Según lo descrito por Francés (2008), las necesidades de la marca no se limitan exclusivamente a la comercialización de los productos. Existen planes estratégicos (evaluación de escenarios, competitividad, ciber-información) que tienen relación con el marketing que ayudan a impulsar a las instituciones, empresas, entre otros; a rediseñar, reimpulsar o mejorar los objetivos y procedimientos operativos, con la intención de desarrollar positivamente su posición en el mercado y ubicarse como propulsores de una marca.

Por su parte, el marketing se le conoce como un sistema que cubre las actividades donde se identifican y estudian aquellas necesidades que tienen los consumidores para poder satisfacerlos de la manera más eficiente y efectiva (Kotler y Armstrong, 2012). También permite el flujo de intercambio de productos y/o servicios con la intención de generar una utilidad para las empresas en beneficio de alcanzar sus objetivos comerciales y empresariales (Taca, 2012). 
Para este tipo de estudio lo cualitativo prevalece, haciendo referencia a la percepción que tienen los individuos hacia este tipo de estrategias (marketing y branding), por ende, se trabajó bajo el modelo fenomenológico-hermenéutico porque la imagen corporativa se puede manifestar en multitud de elementos relacionados con la empresa, y cabe señalar aquellos aspectos relacionados con las marcas o productos y servicios, ya que están íntimamente ligados a las percepciones de la compañía en general (Pintado y Sánchez, 2013), alineándose a las características homogéneas que plantearon las teorías y las investigaciones anteriores, que son esenciales a la hora de dar puntos de vistas relacionados con el tema y sus ejes (HernándezSampieri y Mendoza, 2018). Todo enmarcado dentro de los conocimientos fundamentales sobre neurociencia aplicada al marketing, métodos y técnicas de investigación de mercados, branding, comunicación empresarial, factores que inciden en el comportamiento del consumidor y la aplicación de estos elementos en la planeación estratégica.

Finalmente, la investigación determinó que existe una percepción inadecuada de los servicios que oferta la Cámara de Comercio de Ambato, una de las causas es la amplia variedad de servicios que influyen en que la imagen percibida de la institución sea por un lado positiva pero por otro negativa, dicho esto el posicionamiento de la marca institucional es bajo; aún no se encuentra identificado el perfil del socio actual y potencial, esto da como resultado que la comunicación no sea la adecuada en relación al mensaje y a los medios por donde se llega al público potencial. Bonilla (2017) propone un movimiento desde una perspectiva relacional para una orientación interactiva centrándose en las interacciones de múltiples capas que pueden cruzar ambos ámbitos y medios de comunicación. Mientras que la orientación relacional se enfoca en la comunicación, la orientación interaccional pone énfasis en las relaciones multifacéticas basadas en el reparto dentro y entre las comunidades habilitadas digitalmente. Por lo tanto, en una expansión similar a la transformación de la publicidad a la comunicación integrada de marketing, los vendedores de medios sociales deben ser conscientes de la gran cantidad de posibles restricciones y consecuencias derivadas de la participación en los diferentes lugares de los medios sociales.

Por todo esto, es importante emprender el reconocimiento del impacto positivo que representa la imagen corporativa para reimpulsar sus gestiones administrativas y comerciales mediante la implementación del marketing y todo lo que la planeación estratégica ofrece como herramienta gerencial, para obtener resultados satisfactorios y mantenerse dentro del mercado nacional e internacional.

\section{La imagen corporativa y su importancia}

Uno de los principales objetivos que tienen las organizaciones en general es que su visión, misión y marca se destaque dentro del ámbito de los negocios donde ellos se desenvuelven, sobre todo hoy que se vive un sin número y estresante mercado que obliga que estas empresas se mantengan alertas en todo lo relacionado a la innovación, competitividad y sobre todo marketing (Costa, 2018).

Es por esto que se hace necesario establecer estrategias de mercado que tengan como bandera principal la imagen corporativa para realzar la marca de su (s) producto (s) con la intención de mantener o aumentar los ingresos, así como de disfrutar de los ciertos beneficios de mercado 
y, asegurar el éxito, el emprendimiento y el crecimiento que tiene como meta. Como lo describe Buenaño, Moncayo y Tello (2018), dentro de los principios básicos que ofrece el marketing se encuentra el establecimiento y reconocimiento de la empresa por intermedio de la marca comercial, la importancia que ha generado el tener una imagen corporativa que se encuentre acorde y de la mano con los valores de la organización, permite que los consumidores lleguen de manera directa al negocio o producto.

La calidad, la logística del mercado y el manejo de las redes sociales son factores intrínsecos agregados que se unen para lograr la eficacia de la marca ante cualquier target consumidor (García, Bermúdez, Li y Parra, 2018). Esta marca generalmente viene representada mediante un logo, que a su vez se muestra en una imagen que incluye una serie de valores comerciales que, simplemente con observar su diseño, inmediatamente se acciona en la mente del consumidor su deseo de obtenerlo.

Con relación a esto, para solidificar una imagen corporativa exitosa se debe cumplir con una serie de requisitos que el marketing ofrece (Capriotii, 1999). Dentro de estos requisitos se puede identificar particularidades que se relacionan con la marca tales como: colores, tipos de letras, tamaño, posicionamiento estratégico, entre otros, que se pueden englobar en lo denominado identidad corporativa, como se plantea en la Figura 1.

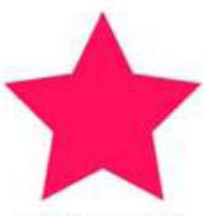

BRANDING

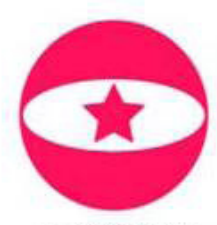

IDENTIDAD

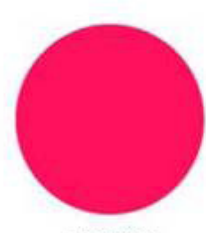

LOGO

Figura 1: Identidad Corporativa

Fuente: Branding corporativo. Gestión estratégica de la identidad corporativa, Molina (2010).

La imagen corporativa define la personalidad de un negocio, donde se engloba desde la identidad de los trabajadores, la apariencia y calidad de los productos, el embalaje o imagen del producto y las características físicas de almacenamiento. Con respecto a la Figura 1, el branding ayuda a los consumidores a elegir los productos adecuados para sus necesidades o preferencias. La marca tiene relación directa generalmente con lo conocido en el marketing como las cuatro $P$ (Precio, Producto, Plaza y Publicidad). Y finalmente el logo es el medio por el cual la empresa se convierte en el ojo referencial del consumidor, pudiendo identificar en el producto la calidad y las bondades comerciales que la marca tiene, simplemente con identificarlo. En definitiva, la imagen corporativa transmite la identidad de la organización mediante la implementación de estrategias unidas al marketing, que impulsan de manera efectiva su condición dentro del mercado frente a los consumidores.

\section{Marketing estratégico y su estructura}

El marketing es considerado como un proceso social y estratégico que emplean las organizaciones 
para resaltar una marca Kotler y Armostrong (2012). Se le denomina social porque intervienen grupos de personas con distintas necesidades, deseos y demandas. Y estratégico porque necesita de elementos básicos, como: la planeación, organización, implementación y el control, para el desarrollo de sus actividades. Entre las características básicas del marketing Stanton, Etzel y Walker (2016) resaltan dos procesos importantes a revisar que se describen en la Figura 2 y en la Figura 3.

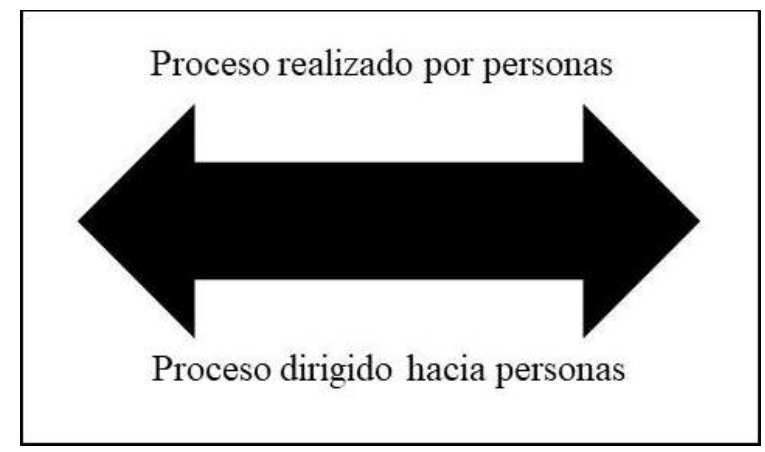

Figura 2. Características del Marketing Básica

Fuente: Fundamentos del marketing, Stanton, Etzel y Walker (2016)

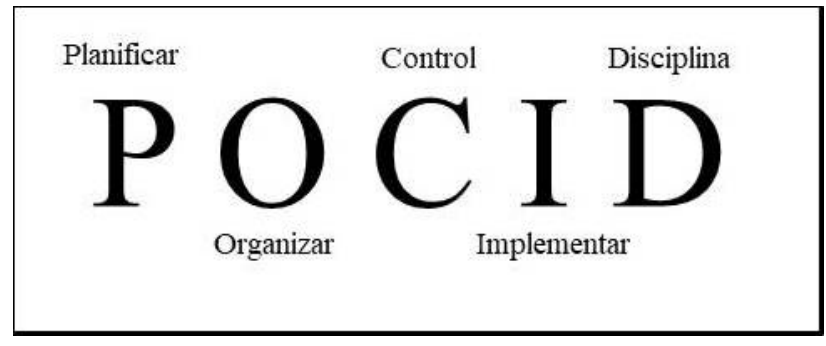

Figura 3. Características del Marketing Secundaria.

Fuente: Fundamentos del marketing, Stanton, Etzel y Walker (2016)

Este proceso de tipo estratégico permite la administración de las ideas productivas que el marketing ofrece para poder planificar, organizar, controlar e implementar el proceso de implementación de la marca desde su inicio hasta su puesta en marcha. Algo interesante que resaltar es la disciplina; la cual permite mantenerse en estado de alerta para no descuidar las etapas del marketing dentro del proceso de implementación, con la intención de lograr el impulso de la organización para posicionar una marca en el mercado global.

A todas estas se afirma que las características del marketing permiten promover los procesos de intercambio en el cual se logra la satisfacción de todas las partes que intervienen en él. Al mismo tiempo, promueve actividades para la identificación de oportunidades, investigación de mercados, formulación de estrategias y tácticas, con el objetivo de desarrollar productos para el crecimiento económico de las organizaciones.

El marketing tiene diferentes atributos que ayudan a la comercialización (Bonilla et al., 2017). Uno de esos atributos del marketing es la identificación de las necesidades y deseos que existen 
en el mercado donde actúa, para retribuirlo en satisfacer al consumidor final con productos o servicios que se transforme al mismo tiempo en una utilidad o beneficio para la organización. Estos productos y servicios están relacionados con alimento, abrigo, sistemas de información, vivienda, seguridad, entre otros, además de otros importantes ítems que tienen relación directa con el marketing psicológico que ataca mediante una marca específica al consumidor final para vender su mercancía.

Vallenilla y Ríos (2014) menciona que existe una clasificación del marketing interesante porque trae consigo factores o elementos que son sumamente importantes para las organizaciones. Esta clasificación se divide en tres grandes grupos. El primero es el marketing 1.0, el cual va direccionada hacia el producto, pero con la intención de llegar a la mente del consumidor. Su estructura permite hacer un buen trabajo de marketing porque llega a la competencia dándole apertura a la innovación como arte de este proceso para ser competente. Cuando se centra en el producto se logra generar más ventas, se entiende mejor el sentido del precio de mercado y se está en contacto directo con la competencia.

La segunda clasificación es el marketing 2.0, que ataca directamente al cliente y llega de manera explícita al corazón del mismo; lo que conlleva a la necesidad de investigar sus preferencias y necesidades de consumo para conocer sus tendencias de compras en el mercado. De esta forma, permite acercarse a él de manera importante. Recordemos que el cliente es la pieza fundamental en todo proceso o modelo de negocio, y la marca, en este caso, es fundamental para conquistarlo ya que el mismo se va convirtiendo en un elemento diferenciador y estratégico para lograr su acercamiento con el producto.

Al estar centrado en el cliente se permite satisfacer las necesidades de mercado, le da un valor agregado al cliente, se apoya en medios tradicionales e interactivos para desarrollar su marca y utiliza las redes sociales para llegar de manera efectiva y eficaz, la publicidad psicológica juega un papel preponderante durante y después del proceso de captación. La tercera clasificación y muy importante es el marketing 3.0, el cual está centrado en los valores organizacionales y su estrategia para transmitirlo de manera efectiva y clara hacia la marca. Lo más resaltante de esta clasificación, es que busca en primer término el cuidado del planeta a pesar de tener como objetivo principal comercial posicionarse en el mercado global. Proteger ese desarrollo sostenible se vuelve bandera con la intención de hacerle ver al mundo la importancia de protegerlo para beneficiarse en el futuro.

Esto se logra por intermedio de programas de creación de un mundo mejor, conocer e inculcar los valores existentes del medio ambiente y mediante el aprovechamiento de los medios interactivos y multidireccionales que permiten el desenvolvimiento y convivencia del desarrollo sostenible junto con el desarrollo comercial. Partiendo de la clasificación anterior, el marketing pudiera ser visto como un sistema de actividades programadas con sentido comercial. Este sistema puede ser como un conjunto de elementos que actúan e interactúan entre sí para lograr objetivos determinados en un periodo de tiempo específico. Este conjunto de elementos es conocido en el mundo del marketing mix gerencial como las 4 P's detalladas en la Figura 4; los cuales coadyuvan al logro de los objetivos comerciales de la empresa (Kotler y Armstrong, 2012). 


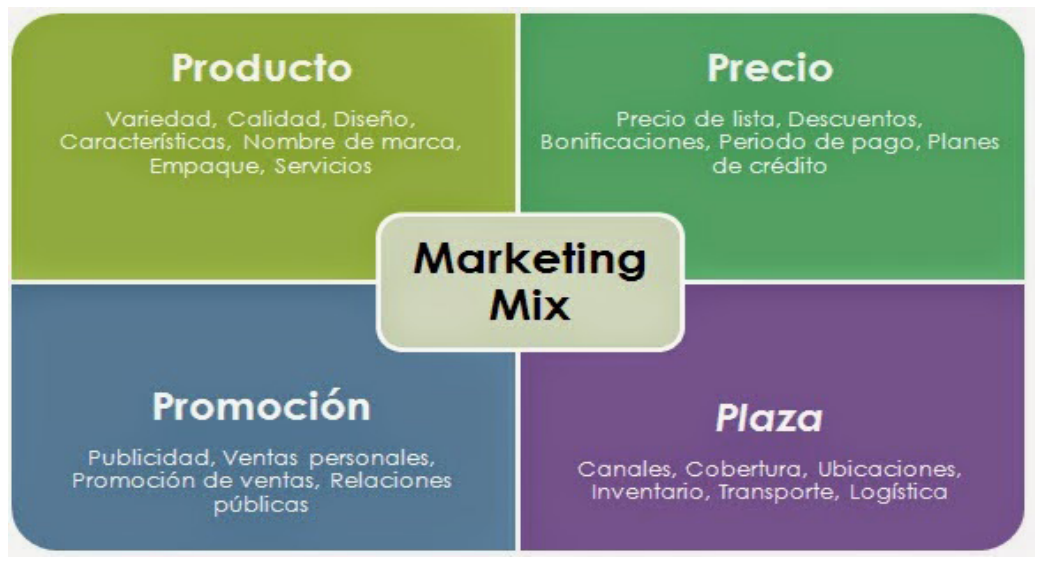

Figura 4. Las 4Ps en la estrategia del marketing.

Fuente: Kotler y Armstrong, 2012.

En base a lo mencionado por Fernández (2015), para desarrollar los elementos arriba señalados, describe que el producto generalmente se le conoce como el conjunto de atributos tangibles 0 intangibles que la empresa ofrece al mercado. Tomando en consideración rasgos que permiten su aceptación tales como: variedad, calidad, diseño, sin descuidar su apariencia externa, el cual, en muchos casos, logra su penetración en el mercado, entre los que se pueden destacar: marca, envase, servicios y garantías.

Generalmente se acude a variables como la publicidad, ventas personales, promociones, cupones y sobre todo relaciones públicas, telemercadeo y propaganda. Como se puede observar, la imagen corporativa tiene que englobar esta serie de elementos y representarlo mediante un logo que permita transmitir lo que se quiere ofrecer al consumidor. Mezclar la mercadotecnia moderna con la imagen se convierte en un elemento táctico importante que proporciona una herramienta valiosa que, durante muchos años, va estructurando los planes de negocio tanto de grandes, medianas como de pequeñas empresas (Muñiz, 2018).

A esto se une los avances tecnológicos que permiten la creación de nuevos escenarios gracias a sus bondades globales, actualizan los procesos apareciendo cada día nuevas propuestas para rediseñar cualquier proceso del marketing mix. Por lo tanto, en caso de presentarse la obsolescencia en el negocio, es importante reinventarse, innovar y revisar los procesos, con el fin de reacondicionar las estrategias con la intención de mantenerse al día con los competidores y lograr a largo plazo la atención de los consumidores finales.

\section{Branding}

La importancia de tener claro los conceptos que se encuentran relacionados, tanto con la imagen corporativa como con el marketing, resulta muy provechoso para entender el éxito del branding en los negocios. La confusión de estos conceptos hace que el significado de branding lo confundan con logo, marca y logística, inclusive como publicidad. A todas estas el branding es conocido como la gestión de todos los activos distintivos de la identidad de una marca sean tangibles e 
intangibles, con el objetivo de construir una promesa y experiencia de marca coherente, diferencial y sostenible en el tiempo (Costa, 2013).

Por lo tanto, el branding no se puede considerar como una estrategia o un proceso de marketing, es más un enfoque de trabajo y una filosofía que se engancha desde la identidad de la marca donde se entrelazan todos los elementos que la empresa necesita proyectar, dentro de los objetivos del branding se destaca la definición clara de la propuesta única de valor o ventaja competitiva, de esta manera queda evidenciado lo que se quiere mercadear. Otro objetivo importante es el diseño de la plataforma de identidad de la marca, que servirá como soporte estratégico que ampare las actuaciones de la organización. Para que sea efectivo lo anterior es necesario emplear una estrategia de marca competitiva que vaya orientada directamente al mercado global y que tenga relación con la línea del marketing y del negocio (Bonilla et al., 2017).

Cabe destacar que, para posicionar la marca en lo conocido como el mapa competitivo, es necesario establecer una línea de comunicación que responda a la plataforma que identifique la marca (Arango, 2014). Finalmente, el branding es considerado como parte de un proceso donde la empresa da a conocer sus diferentes posiciones respecto a la propuesta de valor de manera significativa y diferencial. Funge como un vehículo por donde la visión de la organización tiene que ser percibida y comprendida desde algunos puntos de vista diversos.

La comunicación resulta primordial y se deben integrar todos los trabajadores que conforman la organización, de esta forma se irá conociendo a quién tienen en frente, con quién están tratando, qué es, qué representa y significa, qué hace, cómo lo hace y por qué lo hace (Costa, 2013). Al mismo tiempo, integra las opiniones de los representantes de la empresa para explicar cómo están relacionadas las actividades. Posibilita que aquellas personas que entren en contacto con el producto comprendan sus valores y objetivos organizacionales, y auspicia la consolidación de las ideas que emite la organización de manera que los mismos sean coherentes, eficaces y memorables.

Cuando se habla de planeación estratégica generalmente lo primero que viene a la mente son procesos gerenciales relacionados con las finanzas y el proceso operativo; sin embargo, desde el punto de vista del marketing es importante conocer de qué manera estas estrategias pudieran colaborar de manera exponencial a la imagen corporativa y al producto en general. En primer lugar, existe lo denominado como el juego de los escenarios, que, a pesar de ser netamente gerencial, se puede adaptar y llevar al campo del marketing para aprovechar sus bondades (Francés, 2008).

La esencia del juego de los escenarios viene dada por la proyección de varias propuestas que permitan conocer el verdadero valor de la marca frente a sus competidores y el mercado donde se va a enfrentar (Pintado y Sánchez, 2013). En este mismo orden de ideas, las organizaciones deben tener presente siempre un plan de supervivencia y el crecimiento que permita a largo plazo tener un sentido controlado de las diferentes situaciones presentadas (Forsytgh, 2014). Al mismo tiempo controlar sus oportunidades, sus objetivos y sus recursos por el bien del producto, de esto se trata la planeación estratégica, desarrollar y mantener un plan estratégico dirigido a las metas de la empresa, así también, como a sus capacidades (Leal y Quero, 2014). 
Ahora bien, a nivel organizacional, la gerencia de marketing debe iniciar el proceso de planeación estratégica definiendo su propósito general y su misión. Cabe destacar que en este proceso la misión puede transformar los objetivos iniciales planteados con la intención de reorientar los procesos de reimpulso o lanzamiento de un producto. Es bueno acotar que tanto la planeación de marketing, como la planeación estratégica se apoyan y tienen relación con todas las unidades de negocio de la organización, tratando de catapultar todas las oportunidades de éxito que el marketing puede ofrecer con la intención de rebajar la incertidumbre y aumentar las ganancias a corto y mediano plazo, se detallan los pasos en la planeación estratégica en la Figura 5, Santesmases (2004).

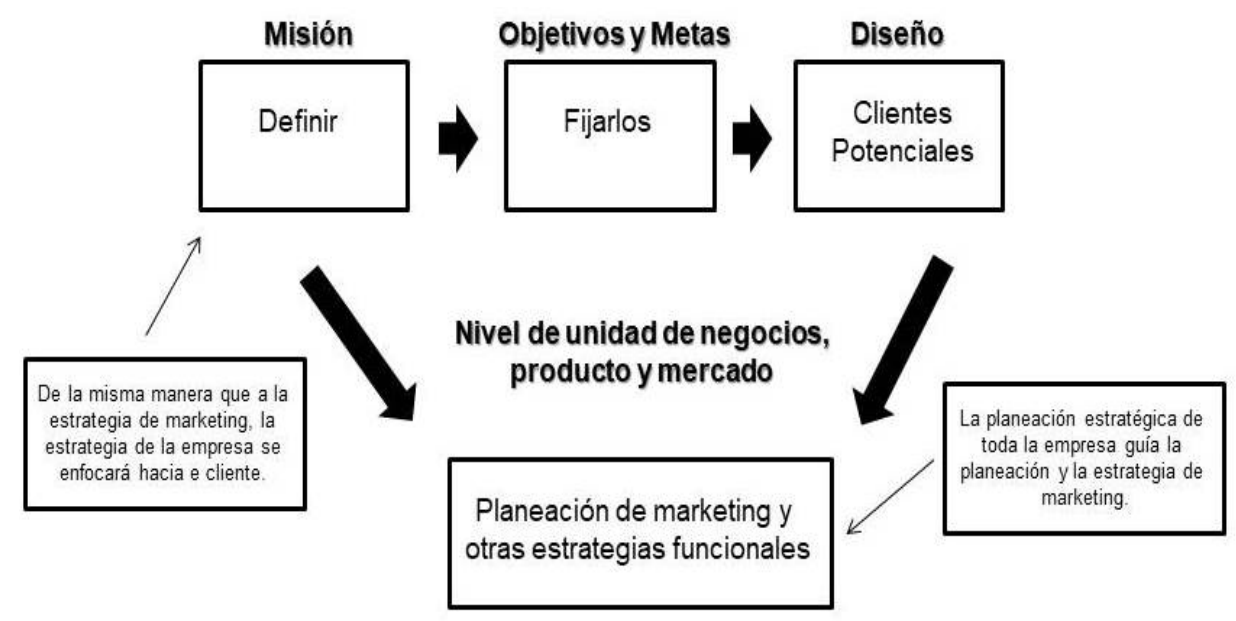

Figura 5. Pasos en la Planeación estratégica.

Fuente: Marketing. Conceptos y estrategias, Santesmanes (2004).

La misión debe ser aprovechada hacia el logro de esos objetivos que resultan de los pasos necesarios para que el enfoque de la planeación estratégica tenga resultados acordes a los esperados. La misión abarca desde lo local hasta lo operativo, y a partir de ese momento el marketing entra en un proceso sumamente interesante como lo es la mezcla del marketing, que no es más que añadir las 4P's del marketing moderno (mix) como lo son: producto, precio, plaza y promoción (Arango, 2014).

\section{Metodología}

La metodología empleada en la investigación tiene un enfoque cualitativo, con diseño fenomenológico, donde se analiza el análisis del discurso de los sujetos en estudio, el método se mezcló con la hermenéutica que sugiere, sin duda, antes que toda otra consideración, un posicionamiento distinto con respecto a la realidad: aquel de las significaciones latentes. Se trata de adoptar una actitud distinta de empatía profunda con las respuestas de los entrevistados, con lo que allí se ha expresado a través del lenguaje. No se trata de suprimir o de intentar inhibir su propia subjetividad (con sus implícitos prejuicios), sino de asumirla, tomando en consideración el tipo de estudio y su influencia interactiva o recurrente (Hernández-Sampieri y Mendoza, 2018). 
La literatura científica recopilada sirvió para extraer una síntesis de teorías que sustentan el tema de investigación, como las ciencias sociales en el área de marketing. Como lo cualitativo generalmente va de la mano con lo inductivo, permitió analizar a profundidad, las diferentes teorías relacionadas con la imagen corporativa como el marketing estratégico. Como población o universo de estudio se escogió a la población de Ambato, se realizó un muestro racimos en donde se seleccionó 12 personas que laboraban en oficina, bajo los siguientes criterios de inclusión: edad entre 20 y 49 años, género masculino y femenino, instrucción desde secundaria a post grado y actividad económica entre empresa pública. Luego se llevó a cabo un focus group, mediante un conversatorio espontáneo e informal, acorde a la técnica elegida se diseñó un cuestionario estructurado con preguntas abiertas (10 preguntas) que posterior mente se envió a 5 expertos todos con el grado de PhD. y con la línea de investigación de ciencias empresariales y administrativas, los informes que ellos dictaminaron sirvieron para que el cuestionario ajuste las preguntas de una manera más precisa que ayude a la investigación a determinar cómo la población percibe la imagen corporativa. Esta validación deja en claro el camino aplanado para que posteriormente se realice una aplicación de este cuestionario a nivel macro donde se puedan calcular índices de confiablidad y validez estadística. El cuestionario sirvió como guía para levantar la información de una manera sistemática y organizada.

Los resultados se presentan con gráficos de una lista agrupada que se utilizó para mostrar grupos y subgrupos de información o pasos y subpasos en una tarea, proceso o flujo de trabajo. El texto de Nivel 1 corresponde a las formas horizontales de nivel superior y el texto de Nivel 2 corresponde a los subpasos verticales bajo cada forma de nivel superior relacionada. Funciona bien para enfatizar subgrupos o subpasos, información jerárquica o múltiples listas de información de la población. Luego se presenta el icono de la institución y todas las respuestas de manera descriptiva haciendo énfasis en las técnicas inicialmente explicadas que permitió concluir objetivamente.

\section{Resultados}

El objetivo de los resultados del estudio permite conocer el criterio de la población de Ambato relacionados con el conocimiento de la marca de la Cámara de Comercio de esa región. De esta forma se determina si hay un conocimiento general sobre la imagen corporativa de la institución, así como la identificación del branding y el reconocimiento de los valores de lo que significa la marca para los habitantes encuestados.

En la Figura 6 se detalla la segmentación del grupo focal. 


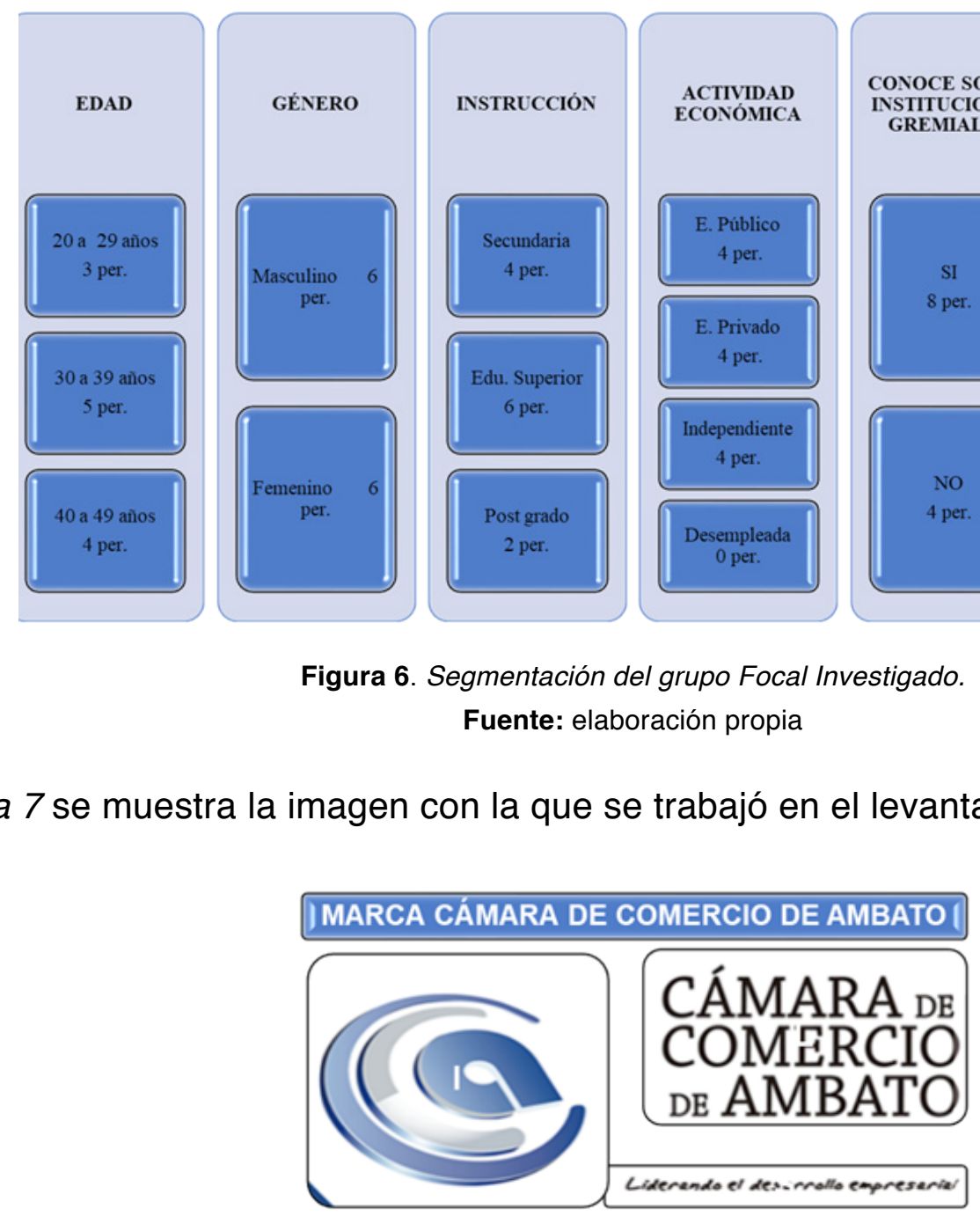

Figura 7. Marca Cámara de Comercio de Ambato.

Fuente: Cámara de Comercio de Ambato

La imagen fue mostrada a los encuestados para conocer su significado, valor, reacción, conocimiento y estética, al indicarles solo el isotipo, la respuesta lo relacionaron con: varios anillos, muchos círculos, una a minúscula en medio y el $50 \%$ de las personas indicaron que ni le gusta ni le disgusta, mientras que el otro $50 \%$ dijo que les gustaba. Lo más resaltante del mismo fue la forma de la a y, lo que menos les gustó, fue que tiene muchas sombras y destellos. En líneas generales y lo más destacable es que ninguno sabía que representa ese isotipo, es decir, a quien representa comercialmente.

Entre otras respuestas encontradas se destaca la combinación de colores el cual fue altamente aceptado y respecto al diseño el $58 \%$ de las personas no les gustó el mismo, el $25 \%$ ni le gustó ni le disgustó y el $17 \%$ restante le gustó completamente. Al parecer el hecho de contener tantas C origina o se inclina hacia la idea de que el mismo representa un centro comercial y no la Cámara de Comercio, entrando un poco de desinformación promocional de la marca. De hecho, y para 
reforzar lo anterior, el $67 \%$ de las personas indicaron que nunca vieron esta imagen antes, el $25 \%$ lo vio en los periódicos y el $8 \%$ en la oficina de la institución, esto ratifica el compromiso de utilizar el branding moderno en conjunto con la planeación estratégica para reforzar esta situación.

Al mostrar el logotipo completo, no relacionaron la imagen con la institución, esto tiene que ver con el diseño tradicional que proyecta un desinterés en la mente del público; así mismo aparece una desvinculación entre la razón social o las actividades económicas que oferta la institución en relación con la filosofía empresarial, es decir, el conjunto de ideas como creencias, principios, prioridades, valores y aspiraciones fundamentales que los empresarios ofrecen para comprender la realidad. Es necesario recordar que la filosofía perdura en el tiempo, existen ejemplos de empresas muy antiguas que han mantenido sus decálogos fundacionales a lo largo de toda su historia. Como Seguros Equinoccial cuyo eslogan es "cumplir la palabra", así como el Banco del Pichincha, su misión es de un "equipo líder que contribuye al desarrollo del Ecuador" otro ejemplo es Produbanco que tiene como filosofía "mantener políticas cuidadosas de manejo de riesgo" y por último se encuentra Diners Club, que se identifica como una tarjeta de crédito "exclusiva, seria, distinta y honesta" Sánchez (2020).

Cuando se refirieron a los colores del logotipo el $67 \%$ de las personas lo consideran sobrio y elegante, mientras el 33\% ni les gustó ni les disgustó, inclusive de ese 33\% la gran mayoría expreso su aspecto oscuro y fúnebre,l y poco refrescante. La tipografía del logotipo no tuvo tanto impacto ya que el $50 \%$ de las personas ni les gustó ni les disgustó, mientras que el otro $50 \%$ les gustó la forma y la posición de las letras. Lo más destacable hasta ahora de todo esto, es que el $67 \%$ de las personas indicaron que nunca vieron esta imagen antes, el $25 \%$ lo observó en los periódicos de circulación y el $8 \%$ en la oficina donde laboran, coincidiendo nuevamente estos números con lo mostrado con el isotipo.

Al compartirles el slogan de la imagen corporativa "Liderando el desarrollo empresarial", la gran mayoría de los encuestados no le encontraron relación con la institución, es más indicaron que les parecía haberlo escuchado antes para otra pauta publicitaria. En referencia al significado del slogan, el $67 \%$ de las personas indicaron que ni les gusta ni les disgusta, mientras que el $33 \%$ indicó que no es original y les disgusta por su falta de originalidad comercial. La tipografía no tuvo aceptación alguna, ya que lo consideran muy enredada e indican que no pueden leerlo con facilidad.

Lo que más les gustó fue la distribución de las palabras y lo que menos le gustó fue el tipo de letra y el significado de la palabra liderando. El color negro les gustó. Lo que le cambiarían es el tipo de letra a una más recta y legible y la palabra liderando. El $67 \%$ de las personas indicaron que nunca vieron esta imagen antes, el $25 \%$ lo vio en los periódicos y el $8 \%$ en la oficina. Una vez vuelve a coincidir tanto con el isotipo como con el logotipo. Algo resaltante durante el levantamiento de la información, los participantes indicaron no tener conocimiento sobre las funciones que realiza la Cámara de Comercio de Ambato. En este sentido, han escuchado hablar de la Cámara de Industrias, Comercio y de la Construcción respectivamente. También sobre la Cámara de Calzado CALTU.

En la Tabla 1 se presenta los servicios que ofrece la Cámara de Comercio de Ambato. 
Tabla 1.

Servicios que ofrece la Cámara de Comercio de Ambato.

\begin{tabular}{ll}
\hline Servicios & Descripción \\
\hline Servicios empresariales & $\begin{array}{l}\text { Enfocados a la capacitación, consultorías y selección de personal, } \\
\text { financiamiento, promoción de negocios nacionales }\end{array}$ \\
\hline Servicios jurídicos & En los ámbitos de arbitraje, mediación y asesoría legal empresarial \\
\hline Servicios sociales & Por medio de la prestación de salas empresariales y de eventos, complejo \\
& deportivo, bolsa de empleo \\
\hline
\end{tabular}

Fuente: elaboración propia

La misión y la visión de la institución se encuentra comprometida y en el olvido organizacional, de hecho, lo primero que se le viene a la mente a los usuarios principales (socios) cuando dicen Cámara de Comercio de Ambato es créditos, cooperativa, salón de eventos, dejando a un lado todos los servicios que la misma trae. Finalmente, de la información recolectada los costos de afiliación son muy elevados, y los mismos no están acordes con los servicios recibidos, presentándose en consecuencia las morosidades de sus integrantes y pérdida de espacio comercial e interés gremial.

\section{Conclusiones}

La importancia que tiene la imagen corporativa para impulsar la marca en las organizaciones se convierte en un instrumento del marketing moderno que toma en consideración elementos que ayudan a posicionarse del mercado donde actúa. La imagen corporativa representa los valores y virtudes de una marca condensada en un diseño que transmita seguridad y deseos de tenerlo para el consumidor.

El branding, se convierte en un elemento complementario en todo proceso donde se encuentre involucrada la marca. Al momento de conjugarse con la imagen corporativa y el marketing moderno permite atacar diferentes ángulos del mercado global abarcando de cierta forma, lo concerniente a la publicidad, la innovación y sobre todo el comportamiento de los competidores. Tener un plan que permita accionar los diferentes cambios del marketing hace posible, en cierta forma, contrarrestar la incertidumbre y atender las necesidades del cliente, aprovechando de cierta forma, la diversidad que la tecnología ofrece en conjunto con las redes sociales.

Con la investigación realizada se evidenció que la Cámara de Comercio de Ambato necesita un plan de marketing para reimpulsar los diferentes servicios que ofrece a la comunidad en general y a sus asociados. Es oportuno, aprovechar las bondades que ofrece la planeación del marketing, para refrescar en primer lugar, su imagen y, con el apoyo de las 4P's de la mercadotecnia, dar a conocer sus servicios que les permita obtener beneficios económicos que solidifiquen sus finanzas. El brandwach de la CCA no tiene la inversión necesaria para conocer los perfiles de los futuros clientes, esto dificulta al marketing estratégico que genera la gerencia. Se debe gestionar nuestra base de datos y recopilar en un solo lugar toda la información que tenemos de nuestros clientes. Es importante porque si trabajamos de manera adecuada podemos acercarnos a ellos y crear 
campañas y estrategias personalizadas en cada punto de contacto con sus clientes: sitios web, landing pages, correo, móvil y redes sociales.

Se identificó los ejes estratégicos del marketing entre los cuales se destacan: el fortalecimiento de la imagen corporativa, el impulso de la gestión administrativa y comercial, la satisfacción de servicio al cliente, el refrescamiento de la cartera de servicios tangibles e intangibles, la comunicación efectiva, entre otros, para el relanzamiento al mercado de la CCA Ambato como pionero Institucional sin fines de lucro. Se deja establecido a futuro que la institución necesita la aplicación de este mismo instrumento a una población más grande para poder generalizar lo que inicialmente se presumió, y es que la población no reconoce o no asocia la imagen corporativa actual con la variedad de servicios, esto da paso a que se investigue las características de los servicios y se vaya creando marcas diferentes para cada una de ellas con un estudio de mercado al target objetivo que tiene la institución. 


\section{Referencias}

Arango, M. (2009). Los diez pilares del mercadeo: Cuando las cuatro P se quedan cortas. Revista Soluciones de Posgrado EIA, 2(3), 161-176. https://revistas.eia.edu.co/index.php/SDP/article/view/306/299

Bonilla, D., Salinas, D., Lalaleo, F., y Velastegui-Hernández, S. (2017). Marketing estratégico en redes sociales de las organizaciones. Un abordaje 360. Revista Científica Ciencia y tecnología, 18(17), 74-85.

Buenaño, D., Moncayo, M., y Tello, F. (2018). Diseño y comunicación visual: perspectivas para su abordaje desde la imagen corporativa. Revista Kepes, 15(17), 251-271. http://doi: 10.17151/kepes.2018.15.17.11

Costa, J. (2013). Los cinco pilares del branding. Anatomía de la marca. Costa Punto Com Editor (CPC).

Costa, J. (2018). Creación de la imagen corporativa: El paradigma del siglo XXI. Revista Razón y Palabra, 22(1), 356-373. https://www.revistarazonypalabra.org/index.php/ryp/article/view/1160

Fernández, V. (2015). Marketing mix de servicios de información: Valor e importancia de la $\mathrm{P}$ de producto. Revista Bibliotecas Anales de Investigación, 11, 64-78. Dialnet-MarketingMixDeServiciosDelnformación-5704542(2).pdf

Forsytgh, P. (2014). Marketing: Las herramientas más novedosas. Ediecuatorial.

Francés, A. (2008). Antología- Debates IESA Competitividad, gerencia y estrategias. Ediciones IESA

García, M., Bermúdez, G., Li, Z. y Parra, F. (2018). Modelo de administración de marcas: Un enfoque teórico basado en la teoría general de sistemas. Revista Esic Market Economics and Business Journal, 49(1), 93-117. https://doi.org/10.7200/esicm.159.0491.1

Hernández, R., Méndez, S., Mendoza, C., y Cuevas, A. (2017). Fundamentos de investigación. McGraw-Hill.

Hernández Sampieri, R. y Mendoza, C. (2018) Metodología de la investigación: Las rutas cuantitativa, cualitativa y mixta. McGraw-Hill.

Kotler, P. y Armstrong, G. (2012) Marketing. Pearson.

Leal, A. y Quero, M. (2014). Manual de Marketing y Comunicación Cultural. Atalaya Proyecto.

Molina, V. (2010). Imagen corporativa. Hermo Grafic.

Muñiz, R. (2018). Marketing en el Siglo XXI. Centro de Estudios Financieros. 
Rivadeneira, M. (2018). Diseño de imagen corporativa y posicionamiento en el mercado para el local de comida rápida "Las Tukas", de la Ciudad de Santo Domingo. [Tesis de Grado]. Universidad Regional Autónoma de Los Andes, Uniandes.

Pintado, T., y Sánchez, J. (2013). Imagen corporativa: influencia en la gestión empresarial. Esic.

Santesmases, M. (2004). Marketing, conceptos y estrategias. Revista Pirámide.

Sánchez, L. (2020). Las 15 empresas top del Ecuador. Revista Gestión. 180(1), 66-98. https://revistagestion.ec/ sites/default/files/import/legacy_pdfs/180_003.pdf

Stanton, W. Etzel, M. y Walker, B. (2016). Fundamentos de marketing. McGraw-Hill

Taca, C. (2012). Fundamentos del marketing. Tesis de Grado. [Biblioteca Universitaria], Universidad del Rosario.

Vallenilla, R. y Ríos, F. (2014). La magia de la marca personal. Revista Debates IESA, 19(3), 26-29. 
